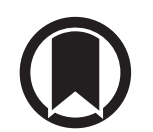

CrossMark

\section{A simple echocardiographic estimate of right ventricular-arterial coupling to assess severity and outcome in pulmonary hypertension on chronic lung disease}

\section{To the Editor:}

The adaptation of right ventricular (RV) systolic function to afterload is a major determinant of outcome in pulmonary hypertension [1]. The gold standard measurement of RV-pulmonary arterial (PA) coupling is the ratio of end-systolic to arterial elastances $\left(E_{\mathrm{es}} / E_{\mathrm{a}}\right)$ which is optimal for RV flow output at minimal energy cost at values between 1.5 and 2 [2]. Progressive RV-PA uncoupling is associated with maintained $\mathrm{RV}$ dimensions down to $E_{\mathrm{es}} / E_{\mathrm{a}}$ values of around 0.8 [3]. Thus, the evaluation of RV-PA coupling would theoretically allow monitoring of the transition from compensated to decompensated RV function in pulmonary hypertension. However, measuring RV-PA coupling at the bedside is technically demanding and invasive. Therefore, simpler imaging surrogates are being evaluated. One of those is the ratio of tricuspid annular plane systolic excursion (TAPSE) as a surrogate of contractility and systolic pulmonary artery pressure (PASP) as a surrogate of afterload, both measured using echocardiography (M-mode for TAPSE and Doppler assessment of the maximum velocity of tricuspid regurgitation for PASP) [4]. The TAPSE/PASP ratio has emerged as a potent predictor of outcome in heart failure [5] as well as in pulmonary arterial hypertension (PAH) [6].

Pulmonary hypertension secondary to chronic lung diseases (PH-LD) is most often mild to moderate, with many patients having a mean pulmonary artery pressure (PAP) $<35 \mathrm{mmHg}$. A small percentage of patients referred for evaluation in dedicated centres may have severe pulmonary hypertension with mean PAP in the range reported in PAH [7]. However, RV function is often altered even in mild-to-moderate $\mathrm{PH}-\mathrm{LD}$, and is an important determinant of survival and functional status in $\mathrm{PH}-\mathrm{LD}$ $[7,8]$. We therefore explored the functional significance and prognostic relevance of the TAPSE/PASP ratio in PH-LD.

We analysed patients with $\mathrm{PH}-\mathrm{LD}$ and idiopathic $\mathrm{PAH}$ (iPAH) enrolled in the prospectively recruiting Giessen PH Registry [9]. The diagnosis of PH-LD was established by a multidisciplinary board before enrolment in the Giessen PH registry [9] between December 2004 and March 2012. Follow-up data were retrieved from the Giessen PH Registry up to February 2018. The analysis included consecutive patients with complete echocardiographic (day 1) and invasive haemodynamic data (day 2) and complete follow-up. The patients with iPAH $(n=193)$ were a subset of a previously reported cohort of 290 patients with PAH [6]. The investigation was approved by the ethics committee of the Faculty of Medicine at the University of Giessen (Approval No. 186/16, 266/11). All participating patients gave written informed consent.

As recently updated [7], PH-LD was defined by a mean PAP of $\geqslant 21 \mathrm{mmHg}(21-24 \mathrm{mmHg}$ with pulmonary vascular resistance (PVR) $\geqslant 3$ Wood Units, or $\geqslant 25 \mathrm{mmHg}$ alone), with mean PAP $\geqslant 35 \mathrm{mmHg}$ alone or mean PAP $\geqslant 25 \mathrm{mmHg}$ with low cardiac index $\left(<2.0 \mathrm{~L} \cdot \mathrm{min}^{-1} \cdot \mathrm{m}^{-2}\right)$ sub-defining severe PH-LD.

@ERSpublications

The ratio of tricuspid annular plane systolic excursion to systolic pulmonary artery pressure is a simple echocardiographic parameter that reflects haemodynamic severity and predicts survival in pulmonary hypertension due to lung diseases. http://bit.ly/2KgLAbR

Cite this article as: Tello K, Ghofrani HA, Heinze C, et al. A simple echocardiographic estimate of right ventricular-arterial coupling to assess severity and outcome in pulmonary hypertension on chronic lung disease. Eur Respir J 2019; 54: 1802435 [https://doi.org/10.1183/13993003.02435-2018]. 
Normally distributed data were expressed as mean \pm SD; non-normally distributed data were expressed as median (interquartile range). Receiver operating characteristic (ROC) analyses and the Youden Index were used to determine thresholds for discrimination of PH-LD severity. Logistic regression models were built to assess the ability of the TAPSE/PASP ratio to discriminate severe and non-severe PH-LD and to predict survival. In all analyses, $\mathrm{p}<0.05$ was considered significant.

In total, 172 patients with PH-LD were included (age: 58 \pm 26 years; mean PAP: 34 (28-41) mmHg; PVR: 5.5 (3.8-7.9) Wood Units; cardiac index: $2.5 \pm 0.7 \mathrm{~L} \cdot \mathrm{min}^{-1} \cdot \mathrm{m}^{-2}$ ). 78 (45.3\%) patients had pulmonary hypertension due to chronic obstructive pulmonary disease ( $\mathrm{PH}-\mathrm{COPD})$, which was classified as severe in $21(12.2 \%)$ patients. The remaining $94(54.7 \%)$ patients had pulmonary hypertension due to interstitial lung disease (PH-ILD), which was classified as severe in $44(25.6 \%)$ patients. The patients with PH-COPD had a reduced forced expiratory volume in $1 \mathrm{~s}\left(\mathrm{FEV}_{1}\right)$ of $51 \pm 23 \%$ predicted and a $\mathrm{FEV}_{1} /$ forced vital capacity $\left(\mathrm{FVC}\right.$ ) ratio of $57 \pm 14 \%$. The $\mathrm{FEV}_{1} / \mathrm{FVC}$ ratio was higher in severe versus non-severe $\mathrm{PH}-\mathrm{COPD}$ while $\mathrm{FEV}_{1}$ was not different $\left(\mathrm{FEV}_{1} / \mathrm{FVC}\right.$ : $63 \pm 12 \%$ pred versus $55 \pm 15 \%$ pred, $\mathrm{p}=0.049 ; \mathrm{FEV}_{1}: \mathrm{p}=0.088$; independent t-test). We found no correlation between $\mathrm{FEV}_{1} / \mathrm{FVC}$ or $\mathrm{FEV}_{1}$ and the TAPSE/PASP ratio in severe and non-severe PH-COPD (data not shown). The patients with PH-ILD had reduced total lung capacity (TLC; $71 \pm 21 \%$ pred) and vital capacity (VC; $61 \pm 22 \%$ pred). Neither parameter differed between severe and non-severe PH-ILD (TLC: $\mathrm{p}=0.699$; VC: $\mathrm{p}=0.838$; independent $\mathrm{t}$-test). There were no correlations between TLC or VC and the TAPSE/PASP ratio in severe and non-severe PH-ILD (data not shown).

In the patients with severe PH-LD, TAPSE/PASP ratios and PVR values were in the same range as those observed in the patients with $\mathrm{PAH}$ and significantly lower and higher, respectively, than those observed in the patients with non-severe PH-LD (figure 1a).

ROC analysis identified a cut-off of $0.26 \mathrm{~mm} \cdot \mathrm{mmHg}^{-1}$ for TAPSE/PASP with a sensitivity of $80.6 \%$ and a specificity of $71.2 \%$ to discriminate between severe and non-severe $\mathrm{PH}-\mathrm{LD}$, which was superior to TAPSE or PASP alone (figure 1b). Logistic multivariate analysis (adjusting for age and sex) showed a significant ability of the TAPSE/PASP ratio (dichotomised at $0.26 \mathrm{~mm} \cdot \mathrm{mmHg}^{-1}$; OR: 9.37; $95 \% \mathrm{CI}$ : 4.56-19.26; $\mathrm{p}<0.001)$ to discriminate the haemodynamic phenotypes. In addition, ROC analysis showed that TAPSE/PASP is also able to discriminate between severe and non-severe PH-COPD and severe and non-severe PH-ILD (figure 1c). This was supported by logistic multivariate analysis (multivariate OR for PH-COPD: 18.60; 95\% CI: 4.45-77.75; $\mathrm{p}<0.001$; and PH-ILD: 8.44; 95\% confidence interval: 3.34-21.36; $\mathrm{p}<0.001)$. Interestingly, the TAPSE/PASP ratio predicted survival in PH-COPD as well as in PH-ILD (figure 1d).

Previous studies reported only mild-to-moderate alterations in lung function tests in patients with severe $\mathrm{PH}$ secondary to COPD $[10,11]$, and better lung function in severe PH-COPD compared with non-severe $\mathrm{PH}-\mathrm{COPD}[12,13]$. This is supported by our data showing a higher $\mathrm{FEV}_{1} / \mathrm{FVC}$ ratio in COPD with severe versus non-severe $\mathrm{PH}$, and agrees with the notion of a predominantly vascular phenotype in these patients [11].

Afterload dependent progression of RV function from adaptation over maladaptation and eventually to failure is determining the symptomatic status and prognosis irrespectively of the underlying pulmonary hypertension subgroup [14]. In the present study, the TAPSE/PASP ratio, as an afterload dependent echocardiographic surrogate of RV-PA coupling, showed its clinical utility in PH-LD. This is in line with the previously described prognostic relevance and association to PVR in PAH [6] as well as in heart failure [15]. However, the adequacy of RV adaptation may vary from one patient to another and depend on the presence of co-morbidities, as has been shown for example in patients with systemic sclerosis $[1,2]$. Nevertheless, the impact of chronic infections or an inflammatory state on RV-PA coupling in PH-LD patients need further investigation.

Limitations to the present findings are the absence of invasive validation of the TAPSE/PASP ratio, the high proportion of patients with severe PH-LD (probably related to the fact that the UGMLC is a national tertiary referral centre for pulmonary hypertension), and the currently unclear therapeutic relevance. Other parameters such as FAC and global longitudinal strain, have not been investigated in our study but might also be associated with contractility/coupling and outcome. In addition, variability in load condition might influence the TASPE/PASP ratio due to its afterload dependency.

In conclusion, the TAPSE/PASP ratio is a straightforward and clinically relevant measurement to differentiate between the haemodynamic phenotypes of patients with PH-LD. The TAPSE/PASP ratio might prove to be an important non-invasive tool for the evaluation of future therapeutic interventions in patients with PH-LD. 
a)

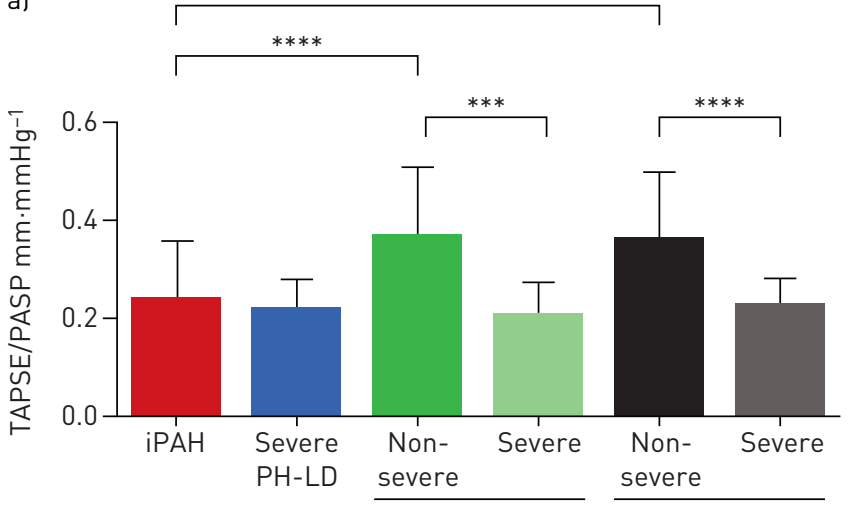

$\mathrm{PH}-\mathrm{COPD}$

$\mathrm{PH}-\mathrm{ILD}$
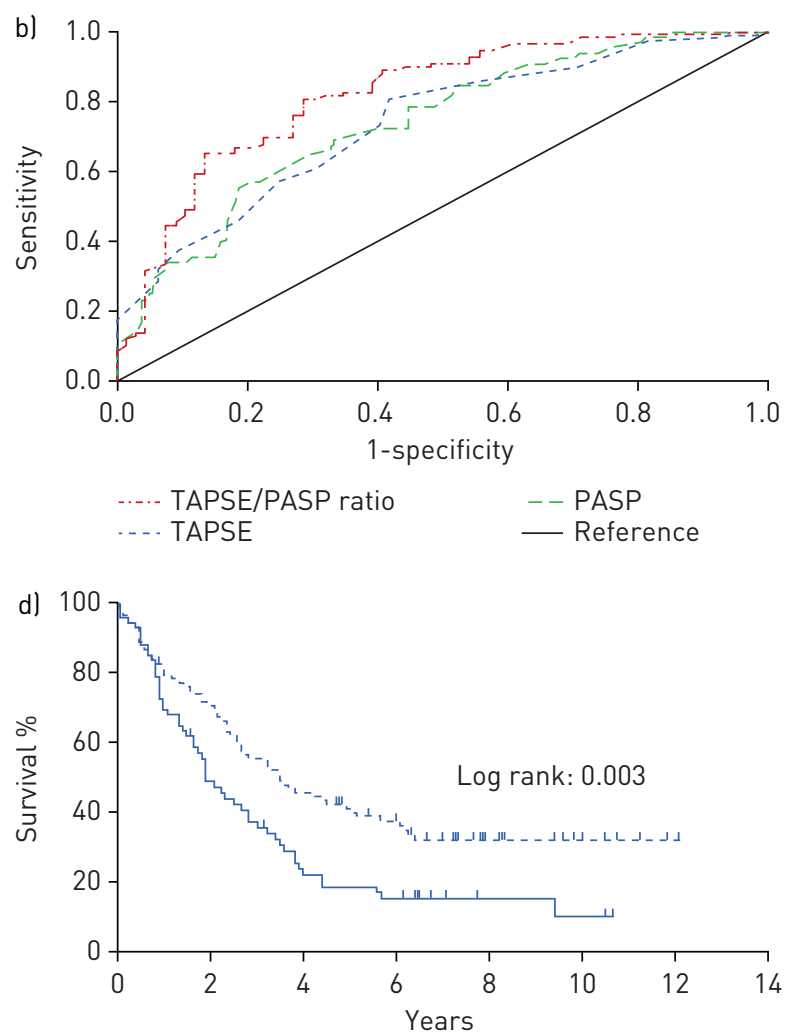

\begin{tabular}{|c|c|c|c|c|}
\hline Numbers at risk & Baseline & 1 year & 3 years & 5 years \\
\hline $\begin{array}{l}\text { PH-LD, TAPSE/PASP } \\
\geqslant 0.26 \mathrm{~mm} \cdot \mathrm{mm} \mathrm{Hg}^{-1}\end{array}$ & 101 & 74 & 51 & 35 \\
\hline $\begin{array}{l}\text { PH-LD, TAPSE/PASP } \\
<0.26 \mathrm{~mm} \cdot \mathrm{mm} \mathrm{Hg}^{-1}\end{array}$ & 71 & 45 & 22 & 11 \\
\hline
\end{tabular}

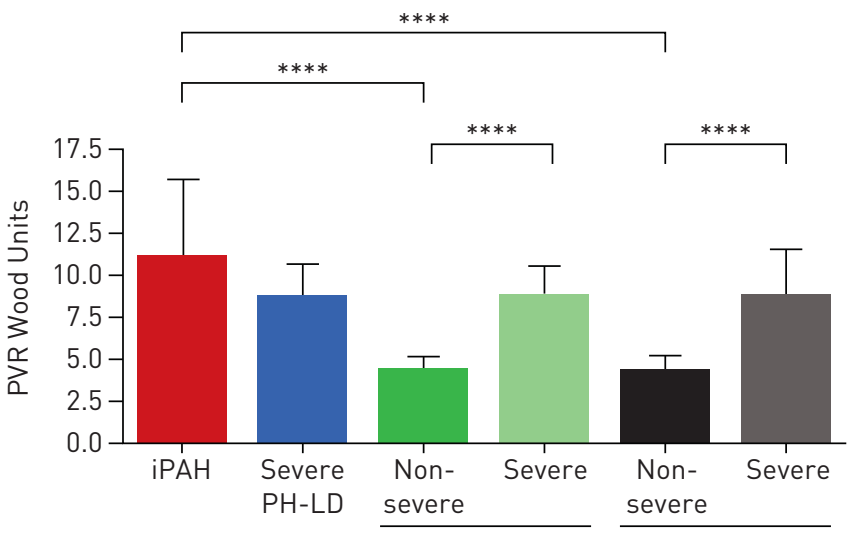

PH-COPD PH-ILD

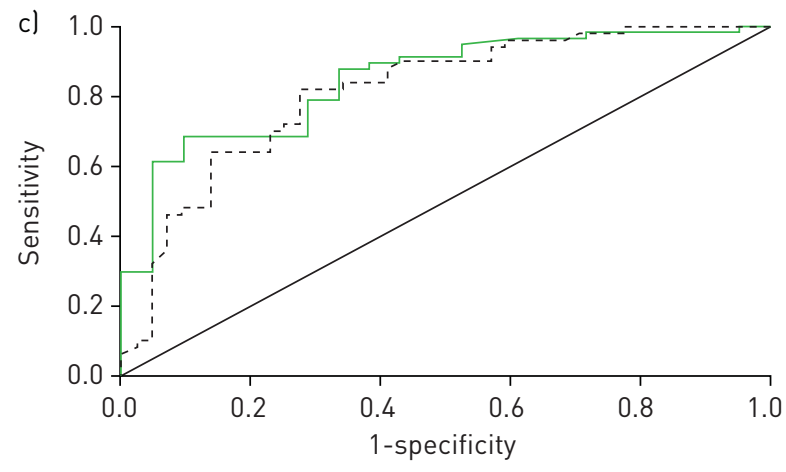

- TAPSE/PASP ratio, PH-COPD - Reference .... TAPSE/PASP ratio, PH-ILD

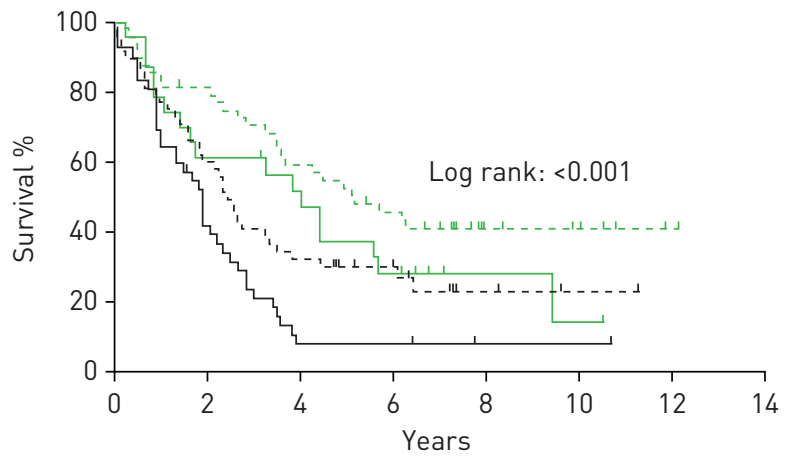

Numbers at risk Baseline 1 year 3 years 5 years

\begin{tabular}{|c|c|c|c|}
\hline $\begin{array}{c}\text { - PH-COPD, TAPSE/PASP } \\
\geqslant 0.26 \mathrm{~mm} \cdot \mathrm{mm} \mathrm{Hg}^{-1}\end{array}$ & 51 & 38 & 32 \\
\hline $\begin{array}{l}\text { - PH-COPD, TAPSE/PASP } \\
<0.26 \mathrm{~mm} \cdot \mathrm{mm} \mathrm{Hg}^{-1}\end{array}$ & 27 & 18 & 14 \\
\hline $\begin{array}{l}\text { - PH-ILD, TAPSE/PASP } \\
\geqslant 0.26 \mathrm{~mm} \cdot \mathrm{mmHg}^{-1}\end{array}$ & 50 & 36 & 19 \\
\hline $\begin{array}{l}\text { PH-ILD, TAPSE/PASP } \\
<0.26 \mathrm{~mm} \cdot \mathrm{mm} \mathrm{Hg}^{-1}\end{array}$ & 44 & 27 & 8 \\
\hline
\end{tabular}

FIGURE 1 The tricuspid annular plane systolic excursion (TAPSE)/ systolic pulmonary artery pressure (PASP) ratio as an indicator of haemodynamic severity and prognosis in pulmonary hypertension secondary to chronic lung disease (PH-LD). a) TAPSE/PASP ratio and pulmonary vascular resistance (PVR) in patients with idiopathic pulmonary arterial hypertension (iPAH) and patients with pulmonary hypertension due to chronic obstructive pulmonary disease (PH-COPD) or interstitial lung disease (PH-ILD) stratified by haemodynamic severity (bar charts show median and interquartile range; ${ }^{* * * *} p<0.0001 ;{ }^{* * *} p<0.001$; between-group differences were analysed with the Kruskal-Wallis test). b) Receiver operating characteristic analyses of the TAPSE/PASP ratio (area under the curve (AUC): 0.825; $p<0.001$ ), TAPSE (AUC: 0.736; $p<0.001$ ) and PASP (AUC: 0.739; $p<0.001$ ) for discriminating between severe and non-severe PH-LD (diagonal segments were produced by ties). $c$ ) Receiver operating characteristic analyses of the TAPSE/PASP ratio for discriminating between severe and non-severe PH-COPD (AUC: $0.847 ; \mathrm{p}<0.001$ ) and PH-ILD (AUC: $0.815 ; p<0.001$ ) (diagonal segments were produced by ties). d) Kaplan-Meier survival curves in patients with PH-LD and subsets with PH-ILD and PH-COPD stratified by the TAPSE/PASP ratio. 
Khodr Tello ${ }^{1}$, Hossein A. Ghofrani ${ }^{1,2,3}$, Charlotte Heinze ${ }^{1}$, Karsten Krueger ${ }^{4}$, Robert Naeije ${ }^{5}$, Christina Raubach ${ }^{1}$, Werner Seeger $\oplus^{1}$, Natascha Sommer ${ }^{1}$, Henning Gall $\oplus^{1,6}$ and Manuel J. Richter ${ }^{1,6}$

${ }^{1}$ Dept of Internal Medicine, Justus-Liebig-University Giessen, Universities of Giessen and Marburg Lung Center (UGMLC), Member of the German Center for Lung Research (DZL), Giessen, Germany. ${ }^{2}$ Dept of Pneumology, Kerckhoff Heart, Rheuma and Thoracic Center, Bad Nauheim, Germany. ${ }^{3}$ Dept of Medicine, Imperial College London, London, UK. ${ }^{4}$ Institute of Sports Science, Department of Exercise and Health, Leibniz University Hannover, Hannover, Germany. ${ }^{5}$ Erasme University Hospital, Brussels, Belgium. ${ }^{6}$ These authors have contributed equally to this work.

Correspondence: Manuel J. Richter, Dept of Internal Medicine, Justus-Liebig-University Giessen, Universities of Giessen and Marburg Lung Center (UGMLC), Member of the German Center for Lung Research (DZL), Klinikstrasse 33, 35392 Giessen, Germany. E-mail manuel.richter@innere.med.uni-giessen.de

Received: 21 Dec 2018 | Accepted after revision: 24 April 2019

Acknowledgements: Editorial assistance was provided by Claire Mulligan (Beacon Medical Communications Ltd, Brighton, UK), funded by the University of Giessen.

Conflict of interest: H.A. Ghofrani reports that this work was funded by the Excellence Cluster Cardio-Pulmonary System (ECCPS) and the Collaborative Research Center (SFB) 1213, Pulmonary Hypertension and Cor Pulmonale, grant number SFB1213/1, project B08 (German Research Foundation, Bonn, Germany), and received editorial support, funded by the University of Giessen; personal fees for consultancy and advisory board work from Bayer and Pfizer, personal fees for consultancy, advisory board work and lectures from Actelion and GSK, personal fees for consultancy from Merck, grants and personal fees for consultancy from Novartis, grants and personal fees for lectures from Bayer HealthCare and Encysive/Pfizer, grants from Aires, German Research Foundation, Excellence Cluster Cardiopulmonary Research and German Ministry for Education and Research, personal fees for advisory board work from Takeda, outside the submitted work. C. Heinze has nothing to disclose. K. Krüger has nothing to disclose. R. Naeije reports grants and personal fees for consultancy and advisory board work from AOPOrphan Pharmaceuticals, Actelion, Bayer, Reata, Lung Biotechnology Corporation and United Therapeutics, outside the submitted work. C. Raubach has nothing to disclose. W. Seeger reports that this work was funded by the Excellence Cluster Cardio-Pulmonary System (ECCPS) and the Collaborative Research Center (SFB) 1213, Pulmonary Hypertension and Cor Pulmonale, grant number SFB1213/1, project B08 (German Research Foundation, Bonn, Germany), and received editorial support, funded by the University of Giessen; personal fees for lectures and consultancy from Pfizer and Bayer Pharma AG, outside the submitted work. N. Sommer reports personal fees from Actelion, outside the submitted work. H. Gall reports that this work was funded by the Excellence Cluster Cardio-Pulmonary System (ECCPS) and the Collaborative Research Center (SFB) 1213, Pulmonary Hypertension and Cor Pulmonale, grant number SFB1213/1, project B08 (German Research Foundation, Bonn, Germany), and received editorial support, funded by the University of Giessen; personal fees from Actelion, AstraZeneca, Bayer, BMS, GSK, Janssen-Cilag, Lilly, MSD, Novartis, OMT, Pfizer andUnited Therapeutics, outside the submitted work. M.J. Richter reports that this work was funded by the Excellence Cluster Cardio-Pulmonary System (ECCPS) and the Collaborative Research Center (SFB) 1213, Pulmonary Hypertension and Cor Pulmonale, grant number SFB1213/1, project B08 (German Research Foundation, Bonn, Germany), and received editorial support, funded by the University of Giessen; grants from United Therapeutics, grants and personal fees for lectures and consultancy from Bayer, personal fees for lectures from Actelion, Mundipharma, Roche and OMT, outside the submitted work. K. Tello reports that this work was funded by the Excellence Cluster Cardio-Pulmonary System (ECCPS) and the Collaborative Research Center (SFB) 1213, Pulmonary Hypertension and Cor Pulmonale, grant number SFB1213/1, project B08 (German Research Foundation, Bonn, Germany), and received editorial support, funded by the University of Giessen; personal fees for lectures from Actelion and Bayer, outside the submitted work.

Support statement: Funded by the Deutsche Forschungsgemeinschaft (DFG, German Research Foundation), Projektnummer 268555672, SFB 1213, Project B08. Funding information for this article has been deposited with the Crossref Funder Registry.

\section{References}

1 Vonk Noordegraaf A, Chin KM, Haddad F, et al. Pathophysiology of the right ventricle and of the pulmonary circulation in pulmonary hypertension: an update. Eur Respir J 2019; 53: 1801900.

2 Vonk Noordegraaf A, Westerhof BE, Westerhof N. The relationship between the right ventricle and its load in pulmonary hypertension. J Am Coll Cardiol 2017; 69: 236-243.

3 Tello K, Dalmer A, Axmann J, et al. Reserve of right ventricular-arterial coupling in the setting of chronic overload. Circ Heart Fail 2019; 12: e005512.

4 Guazzi M, Bandera F, Pelissero G, et al. Tricuspid annular plane systolic excursion and pulmonary arterial systolic pressure relationship in heart failure: an index of right ventricular contractile function and prognosis. Am J Physiol Heart Circ Physiol 2013; 305: H1373-H1381.

5 Guazzi M, Dixon D, Labate V, et al. RV contractile function and its coupling to pulmonary circulation in heart failure with preserved ejection fraction: stratification of clinical phenotypes and outcomes. JACC Cardiovasc Imaging 2017; 10: $10 \mathrm{Pt} \mathrm{B}, 1211-1221$.

6 Tello K, Axmann J, Ghofrani HA, et al. Relevance of the TAPSE/PASP ratio in pulmonary arterial hypertension. Int J Cardiol 2018; 266: 229-235.

7 Nathan SD, Barbera JA, Gaine SP, et al. Pulmonary hypertension in chronic lung disease and hypoxia. Eur Respir J 2019; 53: 1801914.

8 Prins KW, Rose L, Archer SL, et al. Disproportionate right ventricular dysfunction and poor survival in group 3 pulmonary hypertension. Am J Respir Crit Care Med 2018; 197: 1496-1499.

9 Gall H, Felix JF, Schneck FK, et al. The Giessen Pulmonary Hypertension Registry: survival in pulmonary hypertension subgroups. J Heart Lung Transplant 2017; 36: 957-967. 
10 Seeger W, Adir Y, Barbera JA, et al. Pulmonary hypertension in chronic lung diseases. J Am Coll Cardiol 2013; 62: Suppl. 25, D109-D116.

11 Kovacs G, Agusti A, Barbera JA, et al. Pulmonary vascular involvement in chronic obstructive pulmonary disease. is there a pulmonary vascular phenotype? Am J Respir Crit Care Med 2018; 198: 1000-1011.

12 Chaouat A, Bugnet AS, Kadaoui N, et al. Severe pulmonary hypertension and chronic obstructive pulmonary disease. Am J Respir Crit Care Med 2005; 172: 189-194.

13 Boerrigter BG, Bogaard HJ, Trip P, et al. Ventilatory and cardiocirculatory exercise profiles in COPD: the role of pulmonary hypertension. Chest 2012; 142: 1166-1174.

14 Sanz J, Sanchez-Quintana D, Bossone E, et al. Anatomy, function, and dysfunction of the right ventricle: JACC State-of-the-Art Review. J Am Coll Cardiol 2019; 73: 1463-1482.

15 Guazzi M, Naeije R. Pulmonary hypertension in heart failure: pathophysiology, pathobiology, and emerging clinical perspectives. J Am Coll Cardiol 2017; 69: 1718-1734.

Copyright @ERS 2019 